\title{
The Mind-Body Problem Today
}

\author{
Gabriel Vacariu \\ Department of Philosophy, University of Bucharest, Roumania. \\ Email: gvacariu@yahoo.com \\ Received May 31 ${ }^{\text {st }}$ 2011; revised June 25 ${ }^{\text {th }}$, 2011; accepted July $12^{\text {th }}, 2011$.
}

\begin{abstract}
An old philosophical problem, the mind-body problem, has not been yet solved by philosophers or scientists. Even if in cognitive neuroscience has been a stunning development in the last 20 years, the mind-body problem remained unsolved. Even if the majority of researchers in this domain accept the identity theory from an ontological viewpoint, many of them reject this position from an epistemological viewpoint. In this context, I consider that it is quite possible the framework of this problem to be wrong and this is the main reason the problem could not be solved. I offer an alternative, the epistemologically different world's perspective, which replaces the world or the universe. In this new context, the mind-body problem becomes a pseudo-problem.
\end{abstract}

Keywords: Cognitive Neuroscience, Mind, Brain, Epistemologically Different Worlds

\section{Introduction}

In cognitive (neuro) science during the last few decades, as in philosophy in the last centuries, the problem of the mindbody (or mind-brain) phenomena is still open to debate. Paradoxically, since Descartes nobody has proposed a viable alternative view of this problem. Researchers and thinkers have offered some approaches, yet none has gained the assent of the majority of thinkers. Even if most people consider that, ontologically, mind is a physical entity, many of them do not admit the epistemological reduction of the mind to the brain. Thus, all their efforts are towards "saving the phenomena" of the "world": in this case, the phenomena being the mind and the brain. The mind-body problem remains a mystery. From this paradoxical situation, we can draw the conclusion that maybe something is wrong with the problem itself, i.e. with its framework. As it is showed in Vacariu $(2005,2008,2011)$ and Vacariu and Vacariu (2010), this wrong framework is the idea of the "world" or the "universe" (or, as I called, the "unicorn-world"). We have always thought that we exist in a unique world or universe. The unity of that world is the postulation of a single ontological field into which everything has been placed (by "everything” we mean all entities, such as Gods, angels, minds and bodies, planets, tables and microparticles). We can identify this thinking paradigm, this unicorn-world, within the majority of myths, theological doctrines, philosophical approaches, scientific theories, etc. Philosophers and scientists have tried since antiquity to discover the foundations (its structure or its fundamental constituents) of this unicorn-world. The existence of one unique "world" has never been in doubt, even within the multiverse or many worlds approach. However, within this framework, fundamental notions such as "levels", between "brain and mind", emergence, supervenience (and notions from phyiscis like "fundamental particles", the relationships between "microparticles and macroparticles", and the "theory of everything") have remained obscure. The aim of this article is to show that a new framework, the epistemologically different worlds (EDWs), seems to be a better alternative to the mind-brain problem for philosophy and cognitive neuroscience.

\section{The Mind-Body Problem in Cognitive Neuroscience}

Analyzing the mind-body problem there are three elements that need to be taken, epistemologically, into account: the subject, as an observer of both the external world and of internal world; the conditions of observation or conditions of "having something" that include certain external and internal tools of observation; and the observed object or entity. These elements constitute a framework that is not new. Descartes emphasizes the role of perception in identifying two different substances, the mental and the physical. Nevertheless, I would replace the notion of perception with "conditions of observation" for external entities and "conditions of having" for internal entities. In this case regarding the relationship between the subject and the object (external or internal), these notions are equivalent. Usually, when the notion of "perception" is used, we think, immediately, of the sensorial system. However, from my viewpoint, the term "conditions of observation for human beings" stands for conceptual and/or sensorial mechanisms. From one side, with different conditions of observation (that involve different tools of observation), a human being can observe external entities with different structures. The external tools of observation are those instruments or devices that enhance or expand our perceptual mechanisms and help us to perceive external objects. For instance, through perceptual mechanisms, it is possible to observe different parts of a dissected brain. Moreover, expanding these perceptual mechanisms through different devices such as PET or fMRI, certain aspects of neural activation patterns can be observed. From the other side, from my viewpoint, the subject does not perceive any mental states, as Descartes thought (in our days, a remarkable case is Kosslyn (1992) with his mental imagery). Instead, each human "has" certain internal entities like mental representations and processes ${ }^{1}$. The fatal

\footnotetext{
${ }^{1}$ As we will see below (the principle of knowledge), it is better to consider that the mental representations/processes are the "I". The "I" has no spatial dimension; each mental representation involves the entire "I". So, in this case, even the whole-parts relationship is meaningless; we cannot claim that the "I" has a particular mental representation because it is impossible to make a clear difference between the "I" and its mental representations.
} 
consequence for Descartes was that he allocated the mind and body (two ontologically different substances) to the same entity, a human subject or a person ${ }^{2}$. As can be seen below, it is not possible to locate two epistemologically different ontological substances within the same world (as Descartes wanted). In this case, the partition of elements must be preserved: new conditions of observation require new entities within the new worlds, but what kind of worlds? It is not about ontological, many, multiverse or possible worlds, but about "epistemologically different worlds" (EDWs). So, the first principle is this: "Under different conditions of observation, the human subject observes epistemologically different worlds".

If this principle is adopted, it can be assumed that mind and brain (or micro- and macro-particles) belong to epistemologically different worlds. Obviously, it does not mean that changing any condition of observation (or tool of observation), we observe a new $\mathrm{EW}^{3}$. For instance, using different tools of observation (the eyes, fMRI and PET vs. introspection and memory), we can either observe external entities like parts of the brain, patterns of neurons, and neurons or we have internal mental representations and processes. These internal and external entities belong to EDWs. The main mistake for the mindbody (brain) problem has been the placement of the mind and the brain (body) within the same world, the "unicorn-world" 4 . The existence of one unique "world" has never been in doubt (even within the multiverse or many worlds approach). Philosophers and scientists have tried since antiquity to discover the foundations (its structure or its fundamental constituents) of this unicorn-world. However, within this framework, fundamental notions such as "levels", "fundamental particles", the relationships between "microparticles and macroparticles", between "brain and mind", the "theory of everything", and the "essence of things" or "fundamental particles" have remained obscure.

At this point, I would like to bring the ontological dimension into the discussion. "Conditions of observation" have an epistemological dimension, but the idea needs to be extended to the ontological dimension. In order to address the ontological dimension, we replace "conditions of observation" with "conditions of interaction". These notions are equivalent in the sense that every epistemological entity (micro or macro, neural pattern or mental representation, human being or cell) "observes" or interacts with other entities that belong to the same EW. In this sense, it is very important to emphasize that the replacement of the "world" with EDWs entails that we humans are not the only "observers". Each epistemological world (EW) has its own epistemological entities with its own properties and its

\footnotetext{
${ }^{2}$ Fowler emphasizes that Descartes, preserving a traditional relation between doctrine and philosophy, rejects Regius' alternative of the "double-truth option”, i.e., of separating the truth of revelation from the truth of reason. Reaching the stage in which he was aware that the unity between mind and body could not be proved scientifically or philosophically, Descartes pronounced, "the union of mind and body is a reality which escapes philosophical discourse.” (Descartes to Elisabeth, 21 May 1643 in Fowler, p. 385). ${ }^{3}$ In order the subject to observe a new EW, it is necessary a new condition of observation to pass an epistemologic-ontological threshold in relationship with the old condition of observation. (About this threshold and organizational threshold, see Vacariu, 2008).

${ }^{4}$ Another example is the pair table-microparticles. A table (or a planet) is not composed of or is not identical with some microparticles. The table and the microparticles belong to EDWs. The relationship between whole-parts is better understood through the EDWs perspective (Vacariu, 2008; Vacariu \& Vacariu, 2010)
}

own epistemologically different interactions (or epistemologically different laws). However, with the exception of human beings, there are no other entities that can observe/interact with epistemologically different entities from other epistemologically different worlds. Each member of an EW exists only for those entities that belong to that EW alone. I can now introduce the principle of objective reality: "The determining epistemologically different entities and their corresponding constitutive epistemologically different interactions represent the epistemologically different worlds. Each epistemologically different world has the same objective reality."

Mental representation and neural patterns of activation are not the same entity described at different "levels" of description $^{1}$. Without any ontological ground, such "levels” are empty notions, while mental states and neural patterns of activation are epistemologically different entities that belong to EDWs ${ }^{5}$. I emphasize here that the notion of "levels" is completely different then "EDWs". Both "ontological levels" and "epistemological”/“description”/“analysis levels” are erroneous concepts when applied to mind and brain or microparticles and macroparticles! In the first case, we have dualism, in the second there are empty concepts ${ }^{6}$. I emphasize here that, with EDWs, we have the conversion of the ontology into hyperontology that is given by the "constitutive epistemologically different entities". The world or the universe does not exist, it is just created by human imagination. Instead of the "universe", we have the "hyperverse". Nevertheless, hyperverse is an abstract notion; it presupposes a hyperbeing able to observe simultaneously the all EDWs! For actual living beings, this perception is not possible. Under a single set of observational conditions, a subject can observe the constituents of only one EW. Following Bohr, and considering that a subject cannot use two or more tools of observation at the same time, I can postulate the next principle the principle of complementarity: "As human attention is a serial process, the human subject cannot simultaneously observe EDWs."

Regarding the "correlation" (that is, from the EDWs perspective, "correspondence") between a mental state and a neural state there have been several stages in the development of the cognitive neuroscience. I will try to grasp this evolution by analyzing a particular case such as a human subject conscious of seeing, for instance, a red object. If we know that this action produces a mental state to the subject (perception), the question is which is its correspondence within the brain of this subject? In cognitive (neuro) science, three elements have been taken into account for this problem during the last decades.

(a) The activation of a limited number of neural patterns

In the beginning, using fMRI and PET, people from the cognitive neuroscience believed that a perceptual mental state stimulated by an external object was identical with a quite small

\footnotetext{
${ }^{5}$ The EDWs perspective is beyond any kind of relativism. The distinction between the epistemological and ontological dimensions offers me the possibility of avoiding the classic dilemma of relativism. This smooth distinction shows that the EDWs perspective is not based on a circular argument. Epistemologically, the human subject observes and defines the EDWs and its entities in terms of observation but, ontologically, they exist without these processes of observation.

${ }^{6}$ Explaining the difference between objective validity and objective reality in Kant's philosophy, Hanna comments on A239/B298-9 and A248/B305, writing that "empty concepts cannot be meaningfully applied by us either to noumenal objects or to objects of our sensory intuition, and in that sense they are 'impossible'-that is, impossible to use (Hanna, 2001: pp. 90-91).”
} 
neural pattern. They could localized a neural state responsible for a perception of that red object. For instance, Georgopolous shows that we can predict the direction of a monkey's arm movement just before grasping an object by observing the neural patterns activated at that moment. Each neuron "votes" for certain direction and the resulting vector of the neuronal population determines the direction of the arm's movement (Georgopolous, 1988). Rolls points out that "if we know the average firing rate of each cell in a population to each stimulus, then on any single trial we can guess the stimulus that was present by taking into account the response of all the cells" (Rolls, 2001: p. 157). However, in humans even the perceptual awareness is a complex process that implies feed-forward and feedback projections between early visual processing and higher-level neurons. Moreover, probable there are other neural pattern activated in such processes, but actual apparatus of scanning (fMRI, PET, MEG) are not able to grasp them.

In the next stage of the evolution of cognitive neuroscience, one could notice that the red object (with red color, particular texture, etc.) generated the activation of certain neural patterns from different brain areas. The problem (still unsolved) has been the "binding problem": the "correlation" between a red object with several features and the activation of some neural patterns from different areas of the brain. What physical elements and processes correspond to the mental unity of those features? Obviously, the scientific binding problem has mirrored the philosophical mind-body problem. Among the most important approaches for this problem, we recall only two: the "feature integration theory" elaborated by Treisman in the ' $80 \mathrm{~s}^{7}$ (and later developed) (Treisman, 1998, 1999) and the "binding-by-synchrony”. I shall only analyze the latter.

Milner (1974) and Von Der Malsburg (1981) proposed this alternative to the binding problem, while Singer (2007) elaborated certain experimental researches for supporting this approach. Singer considers that the brain is a system with many operations in parallel, without any center of coordination, as the computationalists claim (Fodor, Pylyshyn). Although this alternative is accepted, it still raises some questions. How is it possible for such computations/processes to take place simultaneously in different brain areas in relationship with the coherent perception and action of the human subject? How the signals from sensorial structures are selected and coordinated with the executive ones and how is the information on features binding encoded? The proponents of the binding-by-synchrony think that the coordination mechanism is represented by the synchronized activity of different neural patterns (usually the frequency is $40 \mathrm{~Hz}$ ). This synchronization is correlated with the unity of mental features in a single entity, the object. Certain internal interactions between the neurons produce this synchronization. Neither Singer nor other authors offer an answer to the question "what do these interactions mean?" It seems that this synchronization is a selective reply for attention and consciousness. Singer believes that recent empirical researches prove that synchronization at a large cortical scale (through beta/gamma frequency) is necessary for the sensorial informa-

\footnotetext{
${ }^{7}$ "For experimental psychology researchers, two papers on binding by Anne Treisman in the 1980s set the course for nearly two decades. Treisman's 'feature integration theory' (FIT) became not only the most influential theory of binding, but also the most influential theory of attention (Holcombe, 2009).”
}

tion to reach perceptual consciousness ${ }^{8}$ (Singer, 2007). However, according to Yi Dong et al. (based on very recent experiments on visual mechanism of monkeys) the synchronization does not depend on the binding problem but only on the selectivity of finding the "border-ownership" of an object (Yi et al., 2008). In other words, the synchronization process takes place for the detection of an object's border and not for the binding of the object's features. Evidently, at the time being, we cannot have a final answer to the binding problem. However, more and more experiments using fMRI and PET run in the last years show that a pyramid of neural patterns corresponds even to a simple mental function.

(b) The pyramid of neural pattern with "different grades of activation"

Here we need to introduce some notions from psychology that will be useful in the following chapters. We shall take all these notions from Mandler (1998). She synthesizes these dichotomies in pair-notions: declarative-procedural, accessibleinaccessible, conscious-unconscious, conceptual-sensorimotor, symbolic-subsymbolic, and explicit-implicit (Mandler, 1998: p. 265). These dichotomies are interconnected and partially overlap without being identical (Mandler, 1998: p. 265). The declarative-procedural distinction is based on whether or not the knowledge in question is accessible or inaccessible to consciousness. Procedural knowledge remains inaccessible to consciousness, since we have access only to the effects of procedures, not to procedures themselves. The fact that we use declarative knowledge for gaining procedural knowledge does not entail our having accessibility to procedural knowledge. We are never aware of the details of procedural knowledge by means of which our habituation can increase the performance of our body for some actions. Mandler maintains that we cannot conceptualize and think explicitly about sensorimotor information. This, of course, does not mean that a person is not aware of sensations (qualia) inVolved in perceptual and motor learning (Mandler, 1998: p. 266).

In this context, what does it mean that mental representations and processes belong to the same subject? From my viewpoint, the answer is that the conscious states are "correlated" with the "most activated" neural patterns, while the unconscious states correspond to less activate neural patterns. Baars was among the firsts who initiated the research on the relationship between conscious and unconscious states, and he proposed the concept of the "global workspace theory" (Baars, 2002, 2007). This global workspace represents in fact the consciousness. There are different mental/psychological functions acting in this workspace. Thus, consciousness is an integrative function meaning that it is a "global workspace of integration". (Baars, 2002) These mental functions are correlated with the "inde-

\footnotetext{
${ }^{8}$ Another alternative to the binding problem is that "the perceptual unity is an illusion, with only coordinated behavioral output being in need of an explanation” (Sevush, 2006) However, both approaches have problems. "The evidence for temporal synchrony as a basis for binding has been criticized on both technical and conceptual grounds, while the argument that perceptual unity is an illusion has been challenged both empirically and philosophically." (Sevush, 2006) From my viewpoint, the question is this "perceptual unity" is an illusion for whom? For the "I" that is an illusion, too? Then, we have to return Hume's idea: the self does not exist, it is just an amalgam of biological entities. In searching for the fundamental particles we have extended this idea to all entities that we know in the "universe". So, the conclusion of such Humean framework could be: "Everything that we know (including us) is appearance!” Could we live in such framework? ${ }^{9}$ Baars specifies other important people from the cognitive science who adopted this "global workspace” theory proposed by him.
} 
pendent" functions of the brain ${ }^{10}$ Baars emphasizes that the conscious processes are the product of unconscious processes (Baars \& Franklin, 2007). Thus, cognition is the result of the actions of unconscious processes. For instance, the working memory is achieved through special distributed systems (language components, long term memory, space and temporal framework, etc.) selected by the consciousness. These components are correlated with the widely distributed cortical and subcortical structures (Baars \& Franklin, 2007). The conscious contents are guided or constrained by unconscious contents: the contents of goals, perceptions, conceptual or cultural. For instance, the sentences of words that we think or pronounce are the results of the unconscious processes that form them.

Kanwisher proposes a similar approach (if neural representation is more active then the mental representation correlated with it is consciously active). Kanwisher takes up an idea introduced by Green and Swets according to which perceptual awareness is not "an all-or-none affair, but a graded phenomenon which admits many shades of grey" (Kanwisher, 2001: p. 103). Treisman goes further and claims that attention, i.e. the feed-back projections from high levels to low level of vision, is inVolved even for binding processes ${ }^{11}$ (Treisman, 1998, 1999). For Edelman and Tononi, consciousness is a process that inVolves groups that are widely distributed in the brain (Edelman \& Tononi, 2000). Consciousness mainly presupposes the re-entrant interactions among these groups which are the most important feature of the brain: "reentry leads to the synchronization of the activity of neural groups in different brain maps, binding them into circuits capable of temporally coherent output" (p. 85). Every consciousness state "requires the activation and deactivation of many regions of the brain" (Edelman \& Tononi, 2000: p. 140). Crick and Koch argue that the neural correlates of consciousness at one time engage one part of the cells but their firing influences other neurons, the so-called "penumbra", which makes a contribution to the process of understanding (Crick \& Koch, 2003). In their turn, Llinás and Parre indicate that the "fact that all frequencies are not equal probably determines that certain resonant frequencies will be observed preferentially" (Llinás \& Parre, 1996). In the same line, "The selective property of attention is presumed to be expressed by a positive difference between the activity levels in columns that code for the target and the activity levels in neighboring columns that code for other (distracting) objects (LaBerge, 2002).” To grasp the mind-brain relationship, Merzenich and deCharms introduce the notion of representational perceptual constancy. Constant perceptual representations emerge from the neural level where the pattern of activity of the ensemble of neurons is permanently changing and moving (Merzenich \& deCharms, 1996). Nevertheless, the authors do not explain the origin of this constancy.

The research made with fMRI, PET and MEG in the last years seems to support Baars's approach. For instance, Bartels confirms that large parts of the brain interact for mental proc-

\footnotetext{
${ }^{10}$ The notion of "independence" means that different areas of the brain are responsible for certain mental functions.

${ }^{11}$ The binding problem would correspond-from one viewpoint- to the Kantian notion of synthesis. From EDWs perspective, it is meaningless to search for the binding problem of neural patterns of activation or what the self means from a neural or third-view point. Again, it is like an electron interacts with the table that composed it, i.e., a mixture between EDWs. We have to be aware that the pair "electron-table" is external to a subject, while the pair mind-body involves one external and one "internal” element.
}

esses like attention, binding and segmentation (Bartels, 2009). In other words, a neuronal pyramid is required for the accomplishment of relatively simple mental processes. It seems more and more obvious that any cognitive function activates an entire "neuronal pyramid" but it remains unclear the production of this mechanism and the correlation between a mental function and a neuronal pyramid. The progress of research on this direction suggests that the brain activity is much more complex than we thought in the past. Fodor's modularity, supported by the experiments made by his collaborator Pylyshyn (1999, 2003, 2006), combats Baars' global work space. Modularity means that certain mental parts are specialized in certain mental processes: cognition, perception, motor, etc. Some researchers from the cognitive neuroscience believe that these modules are correlated with certain specialized neural areas. There are not many empirical experiments that support the modularity. However, we mention a recent research on modularity. Using the transmagentic stimulation, Downing tries to prove the visual modular system. Three areas of the visual cortex would play a causal role in the perception of human face, body and various objects (Downing, 2009). But, to understand how a human subject perceives a human face or an object, we have to solve the binding problem. Moreover, such processes involve the consciousness. Thus, the problem becomes more and more complicated: there are complex relationships between the low level (primary visual areas) and the high level (cognition). There are many experiments against the modularity. For instance, there are no constant "correlations" with a particular neuronal area (Haynes, 2009) for a specific feature-the color of an object. The role of the context of color and the experience of human subject in the perception of colors has been acknowledged long time ago. Robertson indicates that a parietal area is essential for the conjunction of certain surface features of an object (Robertson, 2003). But he emphasizes that the binding problem requires other brain areas and processes. (For instance, the high level areas correlated with the attention and the synchronization process.)

(c) The counterpart: the brain and the body

Llinas and Pare write that perception at a given moment is represented by a small percentage of coherently oscillating cellular elements over the whole thalamocortical system. The rest of the thalamocortical system, being silent to such coherence, may in fact represent the necessary counterpart to the temporal pattern of neuronal activity that we recognise individually as cognition (Llinás \& Pare, 1996).

According to these two researchers, we have to add the whole neuronal pyramid with different grades of activation and the rest of the brain to the most activated neural pattern of activation, in order to explain the cognition through the neural areas,. In Baars' paradigm of thinking, I may claim that this "rest of the brain" would correspond to the unconscious/implicit knowledge.

The great majority of researchers from the cognitive science try to explain the cognition (and human behavior) only through the brain processes. However, there are researchers who consider the brain and the body as a unitary system, especially, the proponents of the dynamical system approach, situated cognition, and of the complexity theory. They introduced notions like "embodied" cognition: cognition is the result of the continuous interactions between brain, body and environment. There is 
more or less a theoretical direction of research. Lungarella and Sporns (2006) made an experiment on robotics trying to correlate the intelligence (artificial) with the sensormotor ability and the environment. Sporn was very surprised by these results ${ }^{12}$. It follows that a particular part-the activated pyramidal patterns of neurons - has a counterpart that is the rest of the brain and body. From this perspective, a particular understanding on human subjectivity or human experience is given by the partcounterpart principle: "In physical terms, the part-counterpart relation corresponds to the "I" or the human subjectivity/experience." We can explain the "I" or the human subjectivity/experience (or "What is it like to see a red object") in physical terms only through the part-counterpart relation. Using external tools it is practically impossible to grasp human subjectivity as a whole. Human subjectivity is a universal property of the human species, that is, every human has the feeling of her self, as an individual. However, we cannot perceive this property using external tools. In this context, I emphasize that we cannot make a real difference between the mind and the subjectivity. Perceptions and computations are considered as functions of the mind. Obviously, such functions belong to the "I". In fact, there is no function of the mind that does not belong to the "I". From my viewpoint, the distinction between the mind and the "I" is a pseudo-distinction. As we will see below, the "I" (or the mind) is an EW, and this is the main reason we cannot make a distinction between the "I" and the mind. Therefore, the mind and the "I" are equivalent in this article.

\section{The Human Subjectivity (The “I”)}

In our days the notion of human subjectivity (the mind) is very problematic. The question "Does the 'I' exist?" still has no definitive answer. In contradiction with the EDWs perspective, there is the framework of cognitive (neuro) science in which the researchers try to find the "correlations" between the self and certain neural patterns of activation. For instance, Damasio and Damasio define the self in neural terms: "We see the self as the neural structure and neurobiological states that help us know, without the help of inferences based on language, that the images we perceive are ours rather then somebody else's”.) (Damasio \& Damasio, 1996: p. 22) The subjective state of perceiving an object presupposes different neural structures that represent the image of that object, the image of the self, and the connection between the self and the image of that object, i.e., the convergence zone (p. 25). The "self" means "a collection of images about the most invariant aspects of our organism and its interactions" (p. 23). Offering various experiments from cognitive neuroscience, Macrae et al. try to explain self-knowledge from a neuroscientific viewpoint. They suggest that the medial prefrontal cortex seems to be essential in self-referential and mentalizing processing and social-cognitive functioning (simulation of other minds, the use and representation of social knowledge, and moral reasoning) (Macrae, 2004: p. 1073).

\footnotetext{
12“Really, this study has opened my eyes. I'm a neuroscientist so much of my work is primarily concerned with how the brain works. But brain and body are never really separate, and clearly they have evolved together. The brain and the body should not be looked at as separate things when one talks about information processing, learning and cognition-they form a unit. This holds a lot of meaning to me biologically (Sporns, 2006).” Moreover, in his book from 2008, Fodor recognizes that his mistake was he did not apply the LOT to perception and action (Fodor, 2008).
}

Klein mentions various papers written by different authors that support the idea that "self-descriptiveness produced activation of cortical area associated with semantic memory retrieval (left frontal regions) but not those associated with episodic memory retrieval (right frontal regions)" (Klein, 2004: p. 1080). However, he has a footnote in which he mentions that, even if there are various studies that support the conclusion that the self can be located in the left cerebral hemisphere (that presupposes the doctrine of modularity embraced by cognitive science), neurologically, declarative knowledge is distributed widely across the cortex (p. 1086). This footnote cautions against the location of the self or even self-knowledge in an isolated neural area.

From the EDWs perspective, it is meaningless to check for the correlation between the self and certain neural patterns. If we define the existence of all other epistemologically different entities with the help of their interactions, we can say that the "I" does not interact with anything else. The "I" cannot "observe" itself as a complete entity (in Cartesian terms ${ }^{13}$ ). Moreover, nobody can observe an "I" (in psychological form or the first-person ontology). Does this means that the "I" does not exist? In order to define the existence of human subjectivity, we have to change the notion of the existence. Through the interaction of the brain and the body with the environment, certain patterns of neurons are activated. These brain-body-environment reciprocal causal interactions correspond to sensations and perceptions. Certain implicit knowledge is the "I" or to the mind-EW. However, the mind has no place in the "world", it is just an internal EW or the "I". Because brain, body and environment are in a continuous reciprocal interaction, the neural states and processes are undergoing continuous change. I mention that the implicit knowledge is the results of the development and learning processes throughout the life of each individual. This knowledge corresponds to biological mechanisms that are the results of the evolution of our species and the development of each organism in the continuous reciprocal interactions between brain, body and environment. Such physical interactions correspond to certain mental states and processes. Due to the evolution of species ${ }^{14}$ and development of each individual, the "I" is feelings, desires, etc., I consider the feelings and desire to be knowledge as well. The mental representations and processes (that only correspond to parts of the brain and body) are the "I". Now we can introduce the last principle. Human subjectivity or the self in psychological terms is given by the principle of knowledge: "The "I" is knowledge." I emphasize that, in this case, the content of knowledge has at least four elements that overlap:

1) All knowledge (declarative and procedural, accessible and inaccessible, conscious and unconscious, conceptual and sensorimotor, symbolic and subsymbolic, and explicit and implicit knowledge).

2) All kinds of memory.

3) Descartes' functions. For him, the "I", as a thinking thing, has different functions (or properties) such as doubting, understanding, denying, willing, sensing and imagining (Descartes, 1974: p. 82).

4) Self-knowledge and the capacity (possibility) of knowl-

\footnotetext{
${ }^{13}$ About Descartes' clear, distinct, and complete perceptions, see Vacariu, 2008.

${ }^{14}$ We have to remember Konrad Lorenz who extends Kant's view in explaining that the fit between an organism and its environment is due to evolution (Lorenz, 1941).
} 
edge for manipulating itself. This capacity inVolves, among other features, Fodor's characteristics of the mind: compositionality, systematicity and productivity (Fodor \& Pylyshyn, 1988).

All these elements of knowledge correspond to the biological elements of a human subject. For each self, the elements of knowledge superimpose during the process of development and the adult period to create and change the "I". As a paradox, the "I" is both indivisible and formed by mental states and processes. This indivisibility is equal to the self unity and it represents the possibility of the "I" to access directly (in parallel and not in serial) any parts of it, i.e., any parts of the knowledge. The "I" is the knowledge, i.e., all its mental states and processes, having this ability to access parts of itself. The "I" is the knowledge that can access itself. It is the knowledge acquired by any human subject during the whole life. We are able to survive in a "standard environment" because of this implicit knowledge furnishes the stable status of the "I" that inVolves the "representational perceptual constancy" (Merzenich \& deCharms, 1996; Vacariu, 2005, 2008).

\section{Libet, Llinas and Frith's Approaches and the EDWs Perspective}

In our days, some alternatives from cognitive neuroscience are constructed within an anti-reductionism framework. Many people accept the identity theory ${ }^{15}$ even if some of them use the notion of "correlation" which indicates not a "strict identity" between the mental states and the neural patterns of activity. I would like to analyze three approaches of cognitive (neuro) science (Libet, Llinas, \& Frith) that are, in some aspects, quite close to the EDWs perspective. In fact, I want to show that some assumptions of these approaches would fit better within the EDWs framework than the unicorn-world framework, other assumptions being necessary to be discarded.

I started with Libet's “delay” problem presented by Frith (here, in a short form) and then I analyze Libet's recent notion, the "cerebral mental field" (Libet, 2006). Libet's experiment is related to Helmholtz's “unconscious inferences” (Frith, 2007: pp. 66-68). The subject had to lift her finger whenever she "felt doing so" but at the same time she had to tell Libet "when they 'had the urge' to lift their finger" (Frith, 2007: p. 66). The brain activity was measured with EEG devices in both actions. The urge to lift occurs about $200 \mathrm{msec}$ before the finger is lifted.

\footnotetext{
${ }^{15} \mathrm{~A}$ leader on this trend is Bechtel with his mental mechanisms, decomposition and localization (philosophy of cognitive neuroscience) (Becthel, 2009, 2008). He claims that, using three methods (neuroimages with fMRI, brain's lesions and cell's recording), people from cognitive neuroscience can decompose the mental mechanisms into components parts and functions and then each function is localized within the brain. A leader on the anti- reductionism position (or localization) is Uttal (cognitive neuroscience). Using lesions and image techniques, Uttal considers that we cannot decompose a cognitive system in its components (which cannot be localized) because of the intrinsic inaccessibility of mental processes (Uttal, 2003; Becthel, 2002). (For the debate between these two positions, see Vacariu, 2011) (Against Bechtel's mechanisms and localization, see also Chemero and Silbernstein 2007.) I mention that, in philosophy of mind, the main trend for the last 20 30 years has been an anti-reductionism, some philosophers or even researchers from cognitive science sustain a difference between the mind and the brain, even if both elements are physical matter. For instance, Searle believes that the mind is produced by the brain (Searle, 1992) and Grush (with his emulator theory of representation) assumes certain Cartesian assumptions. (Grush, 2003, 2004).
}

However, the most important thing is that the changes in the brain activity occur about 500 msec before the finger is lifted.

So brain activity indicating that the volunteer was about to lift a finger occurred about 300 msec before that volunteer reported having the urge to lift his or her finger. The implication of this observation is that, by measuring your brain activity, I can know that you're going to have the urge to lift your finger before you know it yourself (Frith, 2007: p. 66).

Frith comprehends that "by measuring your brain activity, I can know that you're going to have the urge to lift your finger before you know it yourself" (p. 66) and thus we believe "we are making a choice when, in fact, our brain has already made the choice. Our experience of making a choice at that moment is therefore an illusion. (p. 67)" The conclusion is that mental events do not take place at the same time with the brain processes. How can we interpret Libet's experiment from an EDWs perspective? Firstly, it confirms the EDWs principle: the mind and the brain belong to EDWs. Moreover, the will of a human subject is a mental process that involves the entire subjectivity of the subject that is the "I". So, we have to follow the principle of knowledge: the "I" is the knowledge. Even when we follow Libet's indication (the subject has to tell Libet when she feels to move her finger), there are other brain areas that become more or less active. Probably, the subject needs large parts of the brain to feel the urge to lift her finger even if the activation of these areas can not be recorded by the actual devices. Obviously, using such measuring instruments for the brain activity, we cannot detect all parts just because the entire "I" is involved. According to the principle of part-counterpart, the "I" corresponds to the brain and body, so we have to insert the whole brain and body into the equation. I believe that we should use the EDWs framework for a better interpretation of Libet's experiment.

Few years ago, Libet introduces another concept, the "cerebral mental field” (CMF), to solve his “delay problem” (Libet, 2006). The CMF is produced by the activity of many neurons. He believes that certain experiments can prove the existence of the CMF. In the analysis of Libet's approach from our perspective, we want to show that it misses the framework of the EDWs. When analyzing the delay necessary to produce certain cerebral neuronal events in relationship with some sensory awareness, Libet concludes that "unconscious cerebral processes precede a subjective sensory experience” (Libet, 2006). This means that all mental states and processes "begin unconsciously", even if these states become conscious or not. Libet strongly underlines that the "features of the CMF can be correlated with brain events, even though the CMF is non-physical, by study of subjective reports from the human subject." In his terms, the "subjective experience (the conscious mind) appears to be a non-physical phenomenon" (Libet, 2006: p. 322)! I believe that, in his attempt to avoid Descartes' dualism ${ }^{16}$, Libet adopts a position very similar to Searle $(1992)^{17}$. Only within this framework, the unicorn-world, they can build their approach and reject all the other approaches ${ }^{18}$. When rejecting

\footnotetext{
16، The CMF is not a Cartesian dualistic phenomenon; it is not separable from the brain.” (Libet, 2006: p. 324).

${ }^{17}$ Mainly, Searle considers that the brain produces the mind (Searle, 1992).

${ }^{18}$ The title of Libet's paper is "Reflections on the interaction of the mind and brain". Obviously, he rejects the identity theory: "Simply stating that some (unknown) configuration of neuronal activities equals consciousness (subjective experience) avoids or begs the problem (Libet, 2006: p. 322).” Nevertheless, only within the unicorn-world, we can presuppose the interactions between the mind and the brain!
} 
Umezawa's "mental field model" that is related to the quantum mechanics (with Bohr and Bohm), Libet asks:

"But this does not solve the problem of how the neuronal activity aspect can also be directly related to the subjective, non-physical aspect of mind. If subjective experience is a non-physical phenomenon, what is it? It should be added that subjective experience also involves an integrative property. That is, although billions of individual nerve cell actions give rise to conscious awareness, the actual experience is a unified one. For example, if you look at any object in your external visual field, it appears as a smoothly organized structure, even though we know that several separate areas in the cerebral visual system are contributing colors, spatial configurations, motion, and meaning (interpretation) to it. This has been termed the "binding"' phenomenon (Libet, 2006: pp. 323-324)."

Evidently, within the same world, we cannot explain the relationship between mind, "billions of individual nerve cells", the unity of "consciousness awareness" and the binding problem. Based on his experiments, Libet mentions a "strange experimentally demonstrated" feature of the CMF:

"Awareness of a sensory event does not appear until up to $0.5 \mathrm{~s}$ after the initial response of the sensory cortex to the arrival of the fastest projection to the cerebral cortex (Libet et al., 1991). (...) But, in spite of the actual delay, the individual perceives the normal sensory stimulus without any appreciable delay beyond that for conduction time of the sensory projection from periphery to sensory cortex (Libet et al., 1979). A further experiment showed that up to $0.5 \mathrm{~s}$ of neural activity had to occur for the actual awareness to appear (Libet et al., 1979). Somehow, the subjective time of the actually delayed awareness appears without delay.”

I believe that this famous "delay" could be easier interpreted through the EDWs perspective. The individual perceives the sensory stimulus without delay because of the "I"'s unity. The changes in the brain activity occur about 500 msec before the action of the body and the awareness of the subject just because the "I" is an EW and the body (brain) and mind are EDWs. The "referral in space" grasps exactly the existence of the two EDWs. The CMR is nothing else but the "I" (an EW) that corresponds to (but not produced by) the brain-body-EW. We may conclude that Libet constructs his approach by unifying the entities/processes that belong to the two EDWs. From a EDWs perspective, we, the human beings (as living entities, in general) could not survive in any environment without this continuity. However, this continuity is just the "I" that is the implicit knowledge and has a unity. Without these two elements, we would be like a sophisticated computer without sentience. The implicit knowledge is the "mortar of higher intelligence". Each thought is part of the "I", so the "I" (or its implicit knowledge) is the "foundation of interconnected ideas and concepts"; the "I" is the transcendental framework of our thoughts. ${ }^{19}$

Within the context of the EDWs perspective, let me analyze Llinás's approach to the relationship between the brain, body and the external world. More precisely, how corporeal movements take place and what is the relation between movement and thinking in Llinás’ opinion (Llinás, 2001). As a monist

\footnotetext{
${ }^{19}$ From Kant's transcendentalism philosophy, the framework of our thought is the transcendental apperception that is a priori to any thought. From a EDWs perspective, the "I" is an EW and our thoughts are entities and processes of this EW. Therefore, the "I" is transcendental in relationship with the thoughts. (For more detail, see Vacariu, 2008).
}

("mind and brain are inseparable”), Llinás defines the mind as one of the "global functional state generated by brain" (Llinás 2001, p. 1). In these expressions, the words "inseparable" and "generated" create problems. "Generated" is quite similar to Searle's notion that reflects the relationship between the mind and the brain (Searle, 1992). However, this framework represents a confusion between two EWDs. From the EDWs perspective, the mind and the brain are not "inseparable", as Llinas claims, but they correspond to one another. Therefore, brain does not "generate" mind at all! It is interesting for me that Llinás includes all "sensorimotor images" and "self-awareness" inside the mind (Llinás, 2001: p. 1). In his book, Llinás argues that the mind "has eVolved as a goal-oriented device that implements predictive/intentional interactions between a living organism and its environment", considering the prediction as the most important mental function (p. 3). Even more interesting it is Llinás' assertion that the mind and the movement of body are strongly related being in fact different parts of the same process. From my viewpoint, Llinás is almost saying that the mind and the body are EDWs! We have to remember, however, that he works within the unicorn world. Only the brain moves the body. Evidently, the mind incorporates all the sensorial and motor images because they simply correspond to the real interactions between the brain, body and the external world.

For Llinás, due to the evolution, the multicellular organisms develop brains necessary for the property of "motricity (p. 15).” Motricity presupposes prediction, and this is one of the most important characteristic of the brain. Prediction and motricity are essential to survive, being the result of an evolution based on trials and errors (Llinás, 2001). Moreover, predictions need to be centralized: "self is the centralization of prediction (p. 23).” From the EDWs perspective, predictions are possible only because of the existence of the self that is knowledge (more exactly, the implicit knowledge) acquired by a person during the entire life. According to Llinas, the "external world and the internal world have different coordinate system reference frames", even if the properties of those two worlds have to be "homomorphic" (Llinás, 2001: p. 64). In the unicorn-world, he is obviously aware of "the differences in coordinate system reference frames between the external and internal worlds and how continuity between perception and execution may/must exist (p. 65)."

Interestingly, we can notice that Llinás is aware about the compulsory unity between "disparate” sensorial inputs, memories and thoughts (that involves the binding problem). For this unity, Llinás bets on the "temporal coherence", the synchronous binding of the activity of individual cells, the neurons (for instance, Llinás, 2001: p. 121). Based on different experimental studies, Llinás indicates the $40-\mathrm{Hz}$ coherent neuronal activity for the temporal coherence. The synchronization makes the transfer from the microscopic to the macroscopic landscape. Nevertheless, we saw above that synchronization is a problematic alternative. In the last paragraph, we have again the problematic notions like "generate" or "binding". These notions presuppose both types of entity (brain and cognition) within the same world. It seems that this is again a mistake. Llinás emphasizes that the self is just the "temporally coherent event that binds, in the time domain, the fractured components of external 
and internal reality into a single construct $(\ldots)^{20}$ (Llinás, 2001: p. 120, his italics)." The self is the centralization of prediction and this centralization is an "abstraction we call the 'self.' (p. 127)" We really do not understand what "abstraction" means. We can only give a meaning to this word if we equalize "abstraction" with an EW! Moreover, "qualia must arise from, fundamentally, properties of single cells, (...) amplified by the organization of circuits specialized in sensory functions. Qualia are that part of self that relates (back) to us! It is a fantastic trick! (Llinás, 2001: p. 226, his italics) Qualia are parts of the self, the mind-EW that is indeed a "fantastic trick" due to the mixture of EDWs! Qualia do not "facilitate the operation" of the brain, qualia are parts of the "I" that is an EW. It is not qualia that furnish a "well-defined" framework for the nervous system necessary for quick decision making, but the "I" that is all qualia and perceptual and cognitive states, i.e., the whole knowledge acquired throughout the life that has the unity absolutely necessary for a living entity to survive. Only by introducing the "I" in such equation, we can explain the human predictions and decisions. For Llinás, there has to be a part of the nervous system that "puts the many segments together into something that beforehand did not exist: a unified whole (Llinás, 2001: p. 226)." We cannot ask about the unity of a table from the viewpoint of an electron! The same state of affair applies to the unity of the "I" and we can say: "Don't ask about this unity from the brain's viewpoint”!

Frith's approach (Frith, 2007) is quite close to the EDWs perspective especially in respect of the mind-body problem. At the end of the prologue, Frith underlines the main idea from his book: the distinction between the brain and the mind is false, the mind is created by the brain. Moreover, the brain creates two illusions in us: (1) "we" have a direct contact with the world (2) our mental world is private. In reality, Frith claims that only the brain has contacts with the external world and not our mind. Again, the brain creates the mind. From a EDWs perspective, mind and life are not the product of the brain or the organism, respectively; they are both EDWs than certain physical entities. The mental world is "private" only as a particular EW not as the product of the brain. In this case, the notion of causality has to be replaced with that of correspondence. Frith strongly believes that the "brain activity is not the same as mental experience” (p. 15) ${ }^{21}$. In other terms, the mind is the product of the brain or the mind emerges from the brain. Frith works within the unicorn-world and it is clear that he misses the framework of EDWs. He assumes a materialist position even if he admits that his approach "sounds" like a dualism (Frith, 2007: p. 23, footnote 5). He tries to convince us that we are not consciousness about most of the neural states and processes, and that the self is the product of the brain. The relationship between brain and mind is not one-to-one. Due to his work inside the unicorn-world and the avoidance of the identity theory, Frith is forced to use such notions like "causation" or "dependence" 22 . Obviously, from the EDWs perspective, the brain is different from the mind but they belong to the EDWs. A subtitle in Chapter 5 tells us directly this idea: "My perception is not of the world, but of my brain's model of the world”! In

\footnotetext{
${ }^{20}$ Or " $(T)$ he binding events comprise the substrate of self (Llinas, 2001: p. 126 , his italics).”

${ }^{21}$ One of his examples is when we see a blue object, we cannot find anything blue in the brain (Frith, 2007: p. 15).
}

my viewpoint, the perception is a mental perception so it belongs to the mind. According to Kant's transcendental philosophy, it is of my "mind's model of the world". Frith argues this idea with the "Ames room" picture, Necker's cube and other visual illusions. The next subtitle, "Perception is a fantasy that coincides with reality”, shows exactly the "correspondences" between the EDWs!

\section{Conclusion}

I consider that philosophy can still have a major role in cognitive (neuro) science in our days just because the philosophical mind-body problem has not been yet solved yet. Any alternative to this problem has created the main framework of working for researchers of cognitive (neuro) science just because major problems of this domain (emergence, reductionism, levels, etc.) are strong related to the mind-brain problem. Working within the unicorn-world, neither the identity theory nor the non- reductionism perspective (ontological or epistemological) is a plausible alternative to the mind-body problem. Mental states could only correspond to entities that belong to other EDWs; they are not identical, produced or supervene on states of other hyperontological status. With the EDWs perspective, we have to move from ontological and/or epistemological frameworks in analysing different classes of entities to a hyperontological framework. Thus, the EDWs perspective rejects the Cartesian dualism, the identity theory and all the nonreductionist approaches and pushes much further Fodor's "special science" framework. If Fodor followed Wittgenstein's philosophy and Carnap's linguistic frameworks (today, we have "conceptual frameworks" or "levels"), the EDWs could offer the (hyper) ontological foundations for the mind-body problem and its related problems through replacing the unicorn-world with the $\mathrm{EDWs}^{23}$.

\section{References}

Baars, J. B. (2002). The conscious access hypothesis: Origins and recent evidence. Trends in Cognitive Science, 6, 47-52. doi:10.1016/S1364-6613(00)01819-2

Baars, J. B., \& Franklin, S. (2007). An architectural model of conscious and unconscious brain functions: Global workspace theory and IDA. Neural Networks, 20, 955-961. doi:10.1016/j.neunet.2007.09.013

Bartels, A. (2009). Visual perception: Converging mechanisms of attention, binding, and segmentation? Current Biology, 19, R300-R302. doi:10.1016/j.cub.2009.02.014

Bechtel, W. (2009). Explanation: Mechanism, modularity, and situated cognition. In P. Robbins, \& M. Aydede (Eds.), Cambridge hand book of situated cognition (pp. 155-170). Cambridge: Cambridge University Press.

Bechtel, W. (2008). Mental mechanisms, philosophical perspectives of

\footnotetext{
${ }^{22}$ Like Libet's and Llinas's positions, Frith's alternative seems to be quite close to Searle's stance ("The rediscovery of the mind", 1992). However, Churchland's critics against Searle's alternative are available to Frith, too. Interesting, more and more thinkers from cognitive science appeal, directly or indirectly, to Searle's approach! The main reason is their framework of the unicorn-world and the rejection of the strict identity between the mind and the brain.

${ }^{23}$ I suggest that, giving up to the paradigm of the "unicorn-world" and working within the hyperverse, we can rethink some problems from physics (entanglement and nonlocality from quantum mechanics or the relationship between Einstein's general theory of relativity and quantum mechanics, hyperspace, etc.). (For this topic, see Vacariu, 2008; Vacariu \& Vacariu, 2010).
} 
cognitive neuroscience. London: Rutledge Taylor \& Francis Group.

Bechtel, W. (2002). Decomposing the mind-brain: A long-term pursuit. Brain and Mind, 3, 229-242. doi:10.1023/A:1019980423053

Chemero, A., \& Silberstein, M. (2007). After the Philosophy of Mind: Replacing Scholasticism with Science. URL (last checked 20 August 2010) http://philsciarchive.pitt.edu/archive/00003200

Crick, F., \& Koch, C. (1997). Towards a neurobiological theory of consciousness. In N. Block, O. Flanagan, \& G. Guzeldere (Eds.), The nature of consciousness (pp. 277-292). Cambridge, MA: MIT Press.

Crick, F., \& Koch, C. (2003). A framework for consciousness. Nature, 6, 119-126.

Damasio, A. R., \& Damasio, H. (1996). Making images and creating subjectivity. In R. Llinas, \& P. S. Churchland (Eds.), The mindbrain continuum: Sensory processes (pp. 19-28). Cambridge, MA: MIT Press.

Descartes, R. (1994). J. Veitch (Translate) A Discourse on method; meditations on first philosophy, principles of philosophy. Indianapolis, IN: Hackett Publishing.

Downing, P. E. (2009). Visual neuroscience: A hat-trick for modularity. Current Biology, 19, R160-R162. doi:10.1016/j.cub.2008.12.037

Edelman, G. M., \& Tononi, G. (2000). Universe of consciousness: How matter becomes imagination. New York, NY: Basic Books.

Frith, C. (2007). How the brain creates our mental world. Oxford: Blackwell Publishing.

Fodor, J. A. (2008). LOT2-The language of thought revisited. Oxford: Oxford University Press.

Fodor, J. A., \& Pylyshyn, Z. W. (1988). Connectionism and cognitive architecture. Cognition, 28, 3-71. doi:10.1016/0010-0277(88)90031-5

Fowler, C. F. (1999). Descartes on the human soul: Philosophy and the demands of Christian Doctrine. Dordrecht, Boston, London: Kluwer Academic Publishers.

Georgopoulos, A. P. (1988). Neural integration of movement: The role of motor cortex in reaching. The FASEB Journal, 2, 2849-2857.

Grush, R. (2003). In defense of some 'Cartesian' assumptions concerning the brain and its operation. Biology and Philosophy, 18, 53-93. doi:10.1023/A:1023344808741

Grush, R. (2004). The emulation theory of representation: Motor control, imagery, and perception. Brain and Behavioral Science, 27, 77-442.

Hanna, R. (2001). Kant and the foundations of analytic philosophy. Oxford: Oxford University Press.

Haynes, J.-D. (2009). Decoding visual consciousness from human brain signals. Trends in Cognitive Sciences, 13, 194-202. doi:10.1016/j.tics.2009.02.004

Holcombe, A. O. (2009). The Binding problem. In E. Bruce Goldstein (Ed.), The sage encyclopedia of perception (preprint). Thousand Oaks: Sage.

Kanwisher, N. (2001). Neural events and perceptual awareness. Cognition, 79, 89-113. doi:10.1016/S0010-0277(00)00125-6

Klein, S. B. (2004). The cognitive neuroscience of knowing one's self. In M. S. Gazzaniga (Ed.), The cognitive neurosciences (pp. 10771089). Cambridge, MA: MIT Press.

Kossylyn, M. S., \& Keonig, O. (1992). Wet mind-The new cognitive Neuroscience. Columbus, $\mathrm{OH}$ : The Free Press.

LaBerge, D. (2002). Networks of attention. In M. S. Gazzaniga (Ed.), Cognitive neuroscience (2nd ed.) (pp. 711-724). Cambridge, MA: MIT Press.

Llinás, R. (2001). I of the vortex: From neurons to self. Cambridge, MA: The MIT Press.

Llinás, R., \& Pare, D. (1996). The brain as a closed system modulated by the senses. In R. Llinas, \& P. S. Churchland (Eds.), The mind-brain continuum: Sensory processes (pp. 1-18). Cambridge, MA: MIT Press.

Libet, B. (2006). Reflections on the interaction of the mind and brain. Progress in Neurobiology, 78, 322-326. doi:10.1016/j.pneurobio.2006.02.003

Lorenz, K. (1941). Kant's doctrine of the a priori in the light of con- temporary biology. In H. Plotkin (Ed.), Learning, development and culture (pp. 121-143). Chichester: John Wiley and Sons.

Lungarella, M. \& Sporns, O. (2006). Mapping information flow in sensorimotor networks. Public Library of Science Computational Biology, 2, 1301-1312.

Macrae, N. C., Heatherton, T. F., \& Kelley, M. W. (2004). A self less ordinary: The medial prefrontal cortex and you. In M. S. Gazzaniga (Ed.), The Cognitive neurosciences (3rd ed.) (pp. 1067-1076). Cambridge, MA: MIT Press.

Mandler, J. (1998). Representation. In W. Damon, D. Kuhn, \& R. S. Siegler (Eds.), Cognition, perception, and language volume 2, Handbook of child psychology (5th ed.) (pp. 255-308). London: John Wiley.

Merzenich, M. M., \& De Charms, C. R. (1996). Neural representations, experience and change. In R. Llinas, \& P. S. Churchland (Eds.), The mind-brain continuum: Sensory processes (pp. 61-82). Cambridge, MA: MIT Press.

Milner, P. M. (1974) A model for visual shape recognition. Psychological Review, 81, 521-535.

Pylyshyn, Z. (1999). Is vision continuous with cognition? The case for cognitive impenetrability of visual perception. Behavioral and Brain Science, 22, 341-365. doi:10.1017/S0140525X99002022

Pylyshyn, Z. (2003). Return of the mental image: Are there really pictures in the brain? Trends in Cognitive Sciences, 7, 113-118. doi:10.1016/S1364-6613(03)00003-2

Pylyshyn, Z. (2006). Imagery. In R. L. Gregory (Ed.), The Oxford companion to the mind (2nd ed.) (pp. 586-587). Oxford: Oxford University Press.

Robertson, L. C. (2003). Binding, spatial attention, and perceptual awareness. Nature Reviews, Neuroscience, 4, 93-102. doi:10.1038/nrn1030

Rolls, E. T. (2001). Representations in the brain. Synthese, 129, 153171. doi:10.1023/A:1013059525140

Searle, J. R. (1992). The rediscovery of the mind. Cambridge, MA: MIT Press.

Singer, W. (2007). Binding by synchrony. Scholarpedia, 2, 1657.

Sevush, S. (2006). Single-neuron theory of consciousness. Journal of Theoretical Biology, 238, 704-725. doi:10.1016/j.jtbi.2005.06.018

Sporns, O. (2006). Good Information? It's not all about the Brain. URL (last checked 27 October 2006) http://www.sciencedaily.com/releases/2006/10/061027081145.htm

Treisman, A. (1998). Feature binding, attention, and object perception Philosophical Transactions of the Royal Society B, 353, 1295-1306. doi:10.1098/rstb.1998.0284

Treisman, A. (1999). Solutions to the binding problem: Progress through controversy and convergence. Neuron, 24, 105-110. doi:10.1016/S0896-6273(00)80826-0

Uttal, W. R. (2002). Response to Bechtel and Lloyd. Brain and Mind, 3, 261-273. doi:10.1023/A:1019944825779

Vacariu, G. (2011). Being and the hyperverse (in English). Bucharest: University of Bucharest Press.

Vacariu, G., \& Vacariu, M. (2010). Mind, life and matter in the hyperverse (in English). Bucharest: University of Bucharest Press.

Vacariu, G. (2008). Epistemologically different worlds. Bucharest: University of Bucharest Press.

Vacariu, G. (2005). Mind, brain and epistemologically different worlds. Synthese Review, 147, 515-548. doi:10.1007/s11229-005-8366-4

Vacariu, G., Terhesiu, D., \& Vacariu, M. (2001). Towards a very idea of representation. Synthese, 129, 275-295. doi:10.1023/A:1013019621505

Von Der Malsburg, C. (1981) The correlation theory of brain function. Internal Report 81-2. Göttingen: Max-Planck-Institute for Biophysical Chemistry.

Yi, D., Mihalas, S., Qiu, F., Von Der Heydt, R., \& Niebur, E. (2008). Synchrony and the binding problem in macaque. Journal of Vision, 8, 1-16, 30. 